\title{
SOX6 and PDCD4 enhance cardiomyocyte apoptosis through LPS-induced miR-499 inhibition
}

\author{
Zhuqing Jia ${ }^{1} \cdot$ Jiaji Wang $^{2} \cdot$ Qiong Shi $^{1} \cdot$ Siyu Liu $^{3} \cdot$ Weiping Wang ${ }^{1} \cdot$ \\ Yuyao Tian ${ }^{1} \cdot$ Qin $\mathrm{Lu}^{1} \cdot$ Ping Chen ${ }^{1} \cdot K_{\text {Kangtao } \mathrm{Ma}^{1} \cdot \text { Chunyan Zhou }}{ }^{1}$
}

Published online: 10 December 2015

(C) The Author(s) 2015. This article is published with open access at Springerlink.com

\begin{abstract}
Sepsis-induced cardiac apoptosis is one of the major pathogenic factors in myocardial dysfunction. As it enhances numerous proinflammatory factors, lipopolysaccharide (LPS) is considered the principal mediator in this pathological process. However, the detailed mechanisms involved are unclear. In this study, we attempted to explore the mechanisms involved in LPS-induced cardiomyocyte apoptosis. We found that LPS stimulation inhibited microRNA (miR)-499 expression and thereby upregulated the expression of SOX6 and PDCD4 in neonatal rat cardiomyocytes. We demonstrate that SOX6 and PDCD4 are target genes of miR-499, and they enhance LPS-induced cardiomyocyte apoptosis by activating the BCL-2 family pathway. The apoptosis process enhanced by overexpression of SOX6 or PDCD4, was rescued by the cardiacabundant miR-499. Overexpression of miR-499 protected
\end{abstract}

Zhuqing Jia and Jiaji Wang contributed equally to this work.

Electronic supplementary material The online version of this article (doi:10.1007/s10495-015-1201-6) contains supplementary material, which is available to authorized users.

Chunyan Zhou

chunyanzhou@bjmu.edu.cn

1 Department of Biochemistry and Molecular Biology, School of Basic Medical Sciences, Beijing Key Laboratory of Protein Posttranslational Modifications and Cell Function, Key Laboratory of Molecular Cardiovascular Sciences, Ministry of Education of China, Peking University, No. 38, Xueyuan Road, Haidian District, Beijing, China

2 Present Address: Beijing Jianhua Experimental School, Yuquan Road 66, Haidian District, Beijing, China

3 Present Address: Department of Epidemiology, Rollins School of Public Health, Emory University, 1518 Clifton Road NE, Atlanta, GA 30322, USA the cardiomyocytes against LPS-induced apoptosis. In brief, our results demonstrate the existence of a miR-499SOX6/PDCD4-BCL-2 family pathway in cardiomyocytes in response to LPS stimulation.

Keywords SOX6 - PDCD4 - LPS - miR-499 . Cardiomyocyte $\cdot$ Apoptosis

\section{Introduction}

Sepsis-induced myocardial functional disorder is one of the main predictors of morbidity and mortality of sepsis [1]; apoptosis is one of the major contributors to the pathophysiology of sepsis [2]. Mediators of sepsis such as lipopolysaccharide (LPS), a gram-negative bacterial cell wall component, triggers apoptosis in cardiac myocytes by promoting the secretion of cytokines such as tumor necrosis factor $\alpha$ (TNF- $\alpha$ ), interleukin-6 (IL-6), IL-10, and interferon (IFN)- $\gamma[3]$.

The SOX (sex-determining region [SRY]-related HMG box) genes were initially identified based on their homology to the HMG box (DNA-binding domain) that is highly similar to that of the SRY protein. The SOX family comprises 20 genes classified into eight groups [4]. SOX6 belongs to the Sox D family, and plays important roles in vertebrate development [5-7]. It also induces apoptosis in esophageal squamous cell carcinoma (ESCC) $[8,9]$. Previously, we demonstrated that SOX6 promoted apoptosis at the late stage of P19CL6 cell cardiac differentiation [10]. However, whether Sox6 is involved in LPS-stimulated cardiomyocyte apoptosis is unclear.

MicroRNAs (miRNAs) exert remarkable effects in diverse apoptosis mechanisms involving SOX6. MiR-16 inhibited apoptosis of ESCC cells by downregulating 
RECK and SOX6 [9]; miR-208 [8] and miR-155 [11] promoted ESCC or hepatocellular carcinoma cell proliferation by targeting SOX6. MiRNAs participate in many physiological and pathophysiological processes of the heart, such as cardiac contraction and morphogenesis, myocardial infarction, and heart failure [12-14]. In the myocardium of rats with acute myocardial infarction, the expression of some miRNAs was altered, including cardiac-abundant miRNAs such as miR-1, miR-133, miR-208, and miR-499 [15-17]. Our previous research demonstrated that miR-499 inhibited apoptosis during cardiac differentiation of P19CL6 cells [10]. MiR-499, which is specifically expressed in skeletal muscle and the heart, was first described in 2005 [18]. Its high expression in the heart indicates that it might participate in some of its physiological and pathophysiological processes. Indeed, it was regulated and functioned differentially in heart development $[6,19,20]$. Whether miR-499 participates in LPSinduced apoptosis is unknown, as is the possible mechanism involved.

In addition, one miRNA may target several genes; one gene may be regulated by more than one miRNA. MiR-499 regulates apoptosis through multi-gene targeting. Previously, we discovered that PDCD4 (programmed cell death 4) was a target of miR-499 in the regulation of hydrogen peroxide $\left(\mathrm{H}_{2} \mathrm{O}_{2}\right)$-induced apoptosis [21], where PDCD4 is upregulated during apoptosis [22]. Subsequently, a number of reports have indicated that it acts as a tumor suppressor and a potential target for anti-cancer therapies [23]. PDCD4 is also involved in the inflammatory response [24, 25] and differentiation [26, 27]. MiR-21 blocked pancreatic $\beta$-cell death [28] and miR-183 suppressed apoptosis in esophageal cancer cells [29] by targeting PDCD4. Currently, whether PDCD4 can be controlled by cardiacabundant miR-499 in LPS-stimulated cardiomyocyte apoptosis is unclear.

In this study, we investigated whether SOX6 and PDCD4, under common control by miR-499, participate in LPS-induced cardiomyocyte apoptosis. LPS stimulation upregulated SOX6 and PDCD4 expression and enhanced cardiomyocyte apoptosis by suppressing miR-499.

\section{Materials and methods}

\section{Cell culture and chemical reagents}

Neonatal rat ventricular myocytes were prepared from 1- or 2-day-old Sprague-Dawley rats as previously described [30]. The cells were cultured in Dulbecco's modified Eagle's medium (DMEM; Gibco, Grand Island, NY, USA) supplemented with $15 \%$ fetal bovine serum (FBS) for $36 \mathrm{~h}$, then the culture medium was changed to serum-free medium and the cells were cultured for another $12 \mathrm{~h}$ before further experiments. H9c2 cells $\left(\mathrm{ATCC}^{\circledR}\right.$ CRL-1446 ${ }^{\mathrm{TM}}$, Manassas, VA, USA) and HeLa cells $\left(\right.$ ATCC $^{\circledR}$ CCL-2 ${ }^{\text {TM }}$ ) were maintained in high-glucose DMEM supplemented with $10 \%$ FBS, penicillin $(100 \mathrm{U} / \mathrm{mL})$, and streptomycin (100 U/mL). LPS (L4391-1MG) was purchased from Sigma-Aldrich (St. Louis, MO, USA) and dissolved in phosphate-buffered saline (PBS).

\section{Plasmid constructs and oligonucleotides}

pCMV-SPORT-Pdcd4 (containing the full-length coding sequence of $P d c d 4$ ) was a gift from Dr. Iwata Ozaki (Health Administration Center, Department of Internal Medicine, Saga Medical School, Saga University). pGL3Pdcd4-3'-UTR was kindly provided by Dr. Giridhar Mudduluru (Department of Experimental Surgery Mannheim/Molecular Oncology of Solid Tumors, Deutsches Krebsforschungszentrum and University Heidelberg). pMIR-REPORT-Pdcd4-3'-UTR was constructed by cloning the $3^{\prime}$-untranslated region $\left(3^{\prime}\right.$-UTR) of PDCD4 from pGL3-Pdcd4-3'-UTR into pMIR-REPORT. pcDNA3.1Sox6 (containing the full-length coding sequence of SOX6) was provided by Dr. Veronique Lefebvre (Case Western Reserve University). pMIR-REPORT-Sox6-3'-UTR and its mutant construct have been described previously [10]. Small interfering RNAs (siRNAs) targeting PDCD4 and SOX6 (Table 1) were commercially synthesized by SigmaAldrich and GeneChem (Shanghai, China), respectively. A scrambled 22-nucleotide (nt) miRNA (negative control [NC]), miR-499 Mimic and miR-499 Inhibitor were obtained from RiboBio (Guangzhou, China).

\section{Luciferase assays}

HeLa cells were plated in $24-w e l l$ plates at $5 \times 10^{4}$ cells/ well $24 \mathrm{~h}$ before transfection. PDCD4 and SOX6 luciferase plasmids (400 ng) and $20 \mathrm{ng}$ control Renilla vector were cotransfected with transfection reagent (Lipofectamine ${ }^{\circledR}$ 2000; Invitrogen, Carlsbad, CA, USA). Lysates were collected $48 \mathrm{~h}$ after transfection, and luciferase activity was measured in triplicate using a dual luciferase assay (Vigorous, Beijing, China).

Table 1 siRNAs used for transfection

\begin{tabular}{ll}
\hline Gene & Sequence $\left(5^{\prime}-3^{\prime}\right)$ \\
\hline Pdcd4 & Sense: GUCUAAAGGU GGAAAGCGUd TdT \\
& Anti-sense: ACGCUUUCCA CCUUUAGACd TdT \\
Sox6 & Sense: CACUUGUCAGUACCAUUCATT \\
& Anti-sense: UGAAUGGUACUGACAAGUGTT \\
\hline
\end{tabular}




\section{Western blotting analysis}

Total protein extracts were obtained with lysis buffer $(150 \mathrm{mM} \mathrm{NaCl}, 10 \mathrm{mM}$ Tris [pH 7.2], $5 \mathrm{mM}$ EDTA, $0.1 \%$ sodium dodecyl sulfate [SDS], $1 \%$ sodium deoxycholate, $1 \%$ Triton X-100) containing protease inhibitor cocktail (Sigma-Aldrich). Proteins were separated by electrophoresis on $8-15 \%$ SDS-polyacrylamide gels, transferred to nitrocellulose membranes, and incubated with the corresponding primary antibodies. PDCD4 (sc27123), BAD (sc-8044), BAX (sc-493), BID (sc-6538), and BCL-xL (sc-8392) antibodies were purchased from Santa Cruz Biotechnology (Santa Cruz, CA, USA); SOX6 (ab64946) antibody was purchased from Abcam (Hong Kong, China); $\alpha$-actinin (A7811) was from Sigma-Aldrich. The membranes were also probed for mouse glyceraldehyde-3-phosphate dehydrogenase (GAPDH) as a loading control. The blots were next incubated with peroxidaseconjugated immunoglobulin G secondary antibody and were developed using an enhanced chemiluminescence kit (Millipore, Billerica, MA, USA).

\section{Quantitative real-time PCR}

Total RNA was isolated with TRIzol (Invitrogen), and $2 \mu \mathrm{g}$ total RNA was reverse-transcribed with random primers for complementary DNA (cDNA) synthesis. The cDNA was used for PCR using specific primers. Transcript levels were normalized to 18S rRNA. The primers are listed in Table 2. Each value represents the average of at least three independent experiments.

\section{TUNEL assay and annexin V/PI staining}

Cardiomyocyte apoptosis was measured using terminal deoxynucleotide transferase dUTP nick end labeling

Table 2 Primers used for quantitative real-time RT-PCR

\begin{tabular}{lll}
\hline Primers & Sequence $\left(5^{\prime}-3^{\prime}\right)$ & Product size $(\mathrm{bp})$ \\
\hline Pdcd4 & F: TGCCCGTGT GGCAGTGTC & 190 \\
& R: TGGCCCACCAACTGTGGTGC & \\
Sox6 & F: CCCCTCTGAACATGGTGGTGGC & 145 \\
& R: TGAGACTGCCCCTGCCGAGT & \\
Bad & F: AAGTCCGATCCCGGAATCC & 106 \\
& R: GCTCACTCGGCTCAAACTCT \\
Bax & F: AGTGATGGACGGGTCCGGGG & 156 \\
& R: GGCGGCTGCTCCAAGGTCAG \\
Bid & F: TCTGAGGTCAGCAACGGTTC & \\
& R: CTCTTGGCGAGTACAGCCAG & \\
Bcl- $x$ L & F: CTGACGCCCTTCACCGCGAG & 235 \\
& R: CAAAGGCATCCCAGCCTCCGT \\
\hline
\end{tabular}

(TUNEL) (Roche Life Science, Indianapolis, IN, USA) according to the manufacturer's protocol. Cells cultured on coverslips in 24-well plates were fixed in $4 \%$ paraformaldehyde. The number of TUNEL-positive cells was counted under a fluorescence microscope. For flow cytometry analysis, briefly, cultured cells were harvested by trypsinization and washed with PBS. Cells $\left(1 \times 10^{6}\right)$ from each sample were processed for annexin $\mathrm{V} /$ propidium iodide (PI) apoptosis detection (Dojindo, Kumamoto, Japan) according to the manufacturer's instructions.

\section{Statistical analysis}

The data are reported as the mean \pm standard deviation. Comparisons were analyzed using Student's $t$ test or ANOVA. $p<0.05$ was considered to indicate statistical significance.

\section{Results}

\section{MiR-499 levels were downregulated in response to LPS stimulation}

Cardiac-abundant miRNAs such as miR-1, miR-133, miR208, and miR-499 regulate diverse aspects of cardiac function, including cardiomyocyte proliferation, differentiation, contractility, and stress responsiveness. To examine their roles in cardiac cell response to LPS stimulation, we treated cardiomyocytes with $1 \mu \mathrm{g} / \mathrm{mL}$ LPS. Short exposure $(6 \mathrm{~h})$ to LPS decreased miR-499 expression, but did not alter expression of the other miRNAs significantly. A known LPSresponsive miRNA, miR-21 [25], was used as the positive control, and showed decreased expression (Fig. 1a). The LPS-induced expression of miR-499 in the cardiomyocytes was concentration- and time-dependent (Fig. 1b, c).

\section{MiR-499 protected cardiomyocytes from LPS- induced apoptosis}

To study whether miR-499 participates in LPS-induced apoptosis, we established overexpression and knockdown systems by transfecting cardiomyocytes with miR-499 Mimic (chemically synthesized fragments with the same sequence as miR-499 that enhance endogenous miR-499 function) or with miR-499 Inhibitor (chemically synthesized fragments with reversed complementary sequence to miR-499 that weaken endogenous miR-499 effects), a scrambled 22-nt miRNA was used as the NC (Fig. 2a). Flow cytometry (Fig. 2b, c) and TUNEL (Fig. 2d, e) were employed to verify apoptotic cell numbers. The Mimictreated cardiomyocytes were less susceptible to $1 \mu \mathrm{g} / \mathrm{mL}$ LPS treated for $6 \mathrm{~h}$, with a lower apoptosis rate than the 
(a)

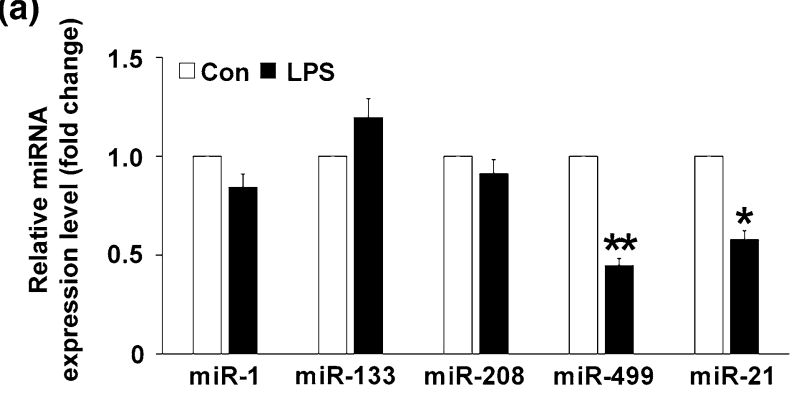

(b)

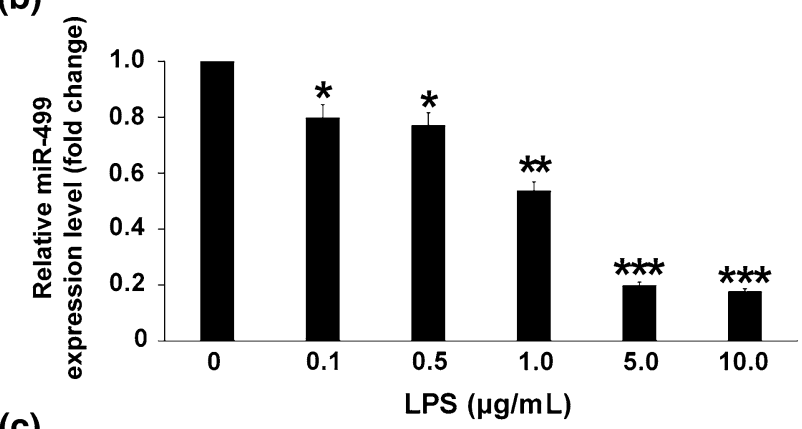

(c)

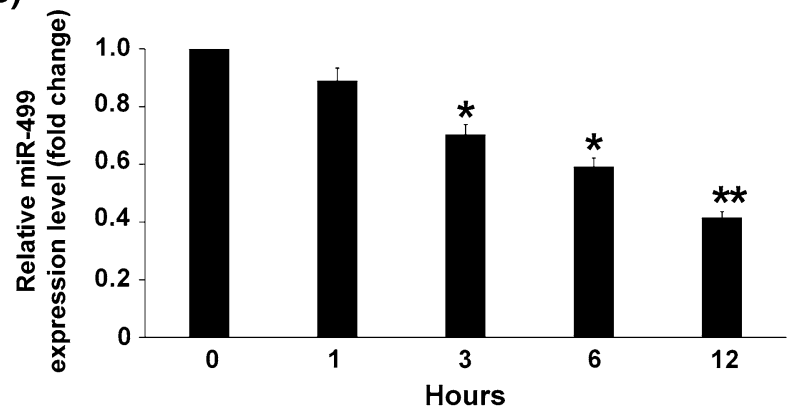

Fig. 1 MiR-499 was downregulated in response to LPS stimulation. a Cardiac-abundant miRNA level in neonatal rat cardiomyocytes exposed to $1 \mu \mathrm{g} / \mathrm{mL}$ LPS at $6 \mathrm{~h}$. Cardiomyocytes not treated with LPS were used as the negative control (con). b MiR-499 level in neonatal rat cardiomyocytes exposed to LPS for $6 \mathrm{~h}$. Cardiomyocytes not treated with LPS $(0 \mu \mathrm{g} / \mathrm{mL})$ were used as the negative control. c MiR499 level in neonatal rat cardiomyocytes exposed to varying durations of $1 \mu \mathrm{g} / \mathrm{mL}$ LPS. Cardiomyocytes not treated with LPS $(0 \mathrm{~h})$ were used as the negative control. Data represent the results of three independent experiments. $* p<0.05,{ }^{* *} p<0.01, * * * p<0.001$ compared with negative control

NC-treated cardiomyocytes; while apoptosis was potentiated in Inhibitor-treated cardiomyocytes compared to the NC-treated group.

Taken together, these results indicate that the miR-499 level is important for maintaining cardiac cell survival in response to LPS stimulation.

\section{SOX6 and PDCD4 participated in LPS-induced cardiomyocyte apoptosis}

To elucidate the molecular mechanisms by which miR-499 regulates apoptosis, we focused on SOX6 and PDCD4, which play important roles in cardiomyocyte differentiation of P19CL6 cells [10] and in protecting adult cardiomyocytes against oxidative stress [21], respectively. SOX6 and PDCD4 expression was first examined to determine whether it is related to LPS-induced cardiomyocyte apoptosis. The results show that SOX6 and PDCD4 expression at both mRNA and protein levels was upregulated in response to $1 \mu \mathrm{g} / \mathrm{mL}$ LPS stimulation (Figs. 3a, b, S1). To explore how SOX6 and PDCD4 are integrated into the cell death program triggered by LPS, the $\mathrm{H} 9 \mathrm{c} 2$ cell line was selected for further study. H9c2 is a cardiac myoblast cell line, similar to primary cardiomyocytes [31]. In response to LPS stimulation, SOX6 and PDCD4 overexpression enhanced the rate of apoptosis from $50 \%$ to almost $80 \%$ (Fig. 3c); knockdown of either SOX6 or PDCD4 decreased the rate of apoptosis from approximately 50-25\% (Fig. 3d). However, without LPS stimulation, the overexpression of SOX6 and PDCD4 only slightly increased the apoptosis rate from 6 to $9 \%$ or $13 \%$ respectively (Fig. S2a), while knockdown of either SOX6 or PDCD4 did not affect the apoptosis rate (Fig. S2b), indicating that the effect of SOX6 and PDCD4 is more significant under the circumstance of LPS-induced damage.

Taken together, our observations suggest that SOX6 and PDCD4 promote LPS-mediated apoptosis.

\section{SOX6 and PDCD4 were targets of miR-499 in LPS- induced apoptosis}

To investigate whether miR-499 affects the expression of the endogenous target genes, we analyzed the SOX6 and $P D C D 4$ sequences and generated luciferase reporters with the $3^{\prime}$-UTR of SOX6 and PDCD4, and the constructs contained a mutated segment of SOX6 (seed sequence AGUCUUA was mutated to AGUCĆUA) and PDCD4 (seed sequence AGUCUUA was mutated to AGUCUGC), respectively (Fig. 4a). As shown in Fig. 4b, c, miR-499 Mimic reduced luciferase activity significantly compared to the NC, whereas no effect was observed with the mutant constructs. This effect was specific because there was no change in luciferase reporter activity when the NC was cotransfected with each reporter construct. Meanwhile, Western blot analysis indicated that miR-499 Mimic attenuated SOX6 and PDCD4 expression, whereas miR499 Inhibitor elevated it (Fig. 4d). These results suggest that SOX6 and PDCD4 both are direct miR-499 targets.

To investigate whether miR-499 inhibited cardiomyocyte apoptosis by suppressing SOX6 and PDCD4, we performed a rescue experiment in miR-499-treated cells by transfecting pcDNA3.1-Sox6 or pCMV-SPORT-Pdcd4 plasmids. Because pcDNA3.1-Sox6 and pCMV-SPORT$P d c d 4$ constructs do not contain $3^{\prime}$-UTR in which the miR499 binding sites locate, they were not targeted by 
(a)

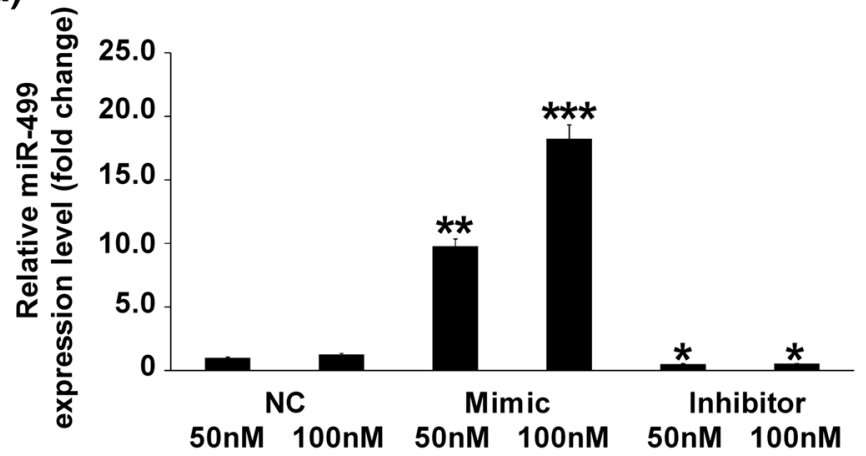

(d)

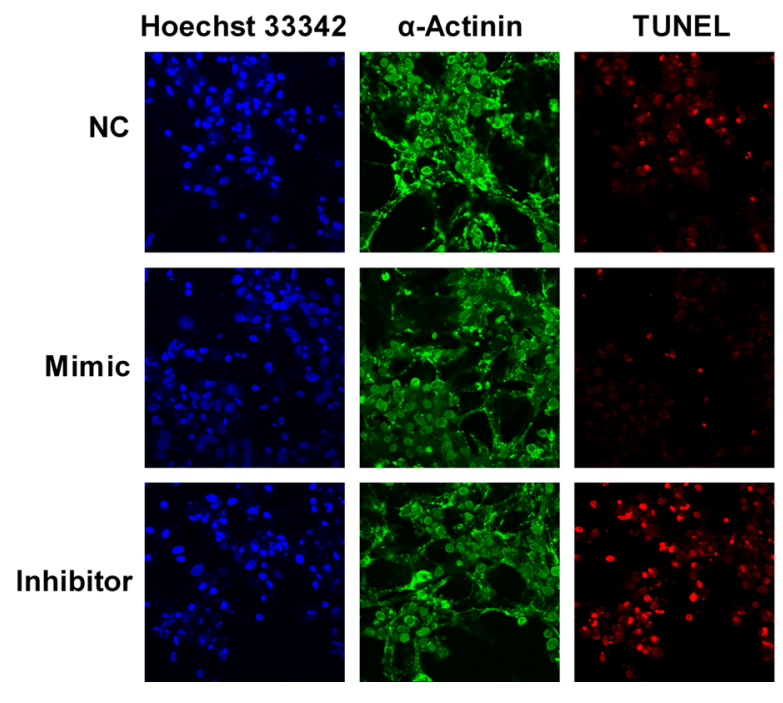

Fig. 2 MiR-499 protected cardiomyocytes from LPS-induced apoptosis. a MiR-499 level in cardiomyocytes after 48-h transfection with miR-499 Mimic or Inhibitor. b Representative flow cytometry images of annexin V/PI-stained cardiomyocytes treated with miR-499 Mimic or Inhibitor, and then exposed to $1 \mu \mathrm{g} / \mathrm{mL}$ LPS for $6 \mathrm{~h}$. c Quantitative results for annexin V-positive cardiomyocytes from three independent experiments. d Representative images of TUNEL-stained

miR-499. Therefore, Western blot showed that SOX6 and PDCD4 protein level was highly upregulated (Fig. 4e).

\section{miR-499 activated the SOX6 and PDCD4 pathways}

BCL-2 family members are major regulators of mitochondrial integrity and mitochondria-initiated caspase activation. The BCL-2 family has both anti-apoptotic and pro-apoptotic members, and act as the downstream key factors of many apoptosis mediators [32]. To investigate how SOX6 and PDCD4 regulate LPS-induced cardiomyocyte apoptosis, pcDNA3.1-Sox6 and pCMV-SPORT$P d c d 4$ overexpression plasmids as well as their specific siRNAs were used to transfect H9c2 cells. Western blotting indicated that the overexpression plasmids and specific (b)

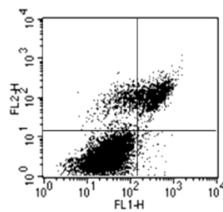

Negative Control

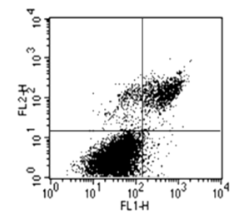

Mimic

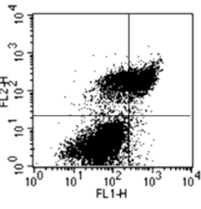

Inhibitor (c)

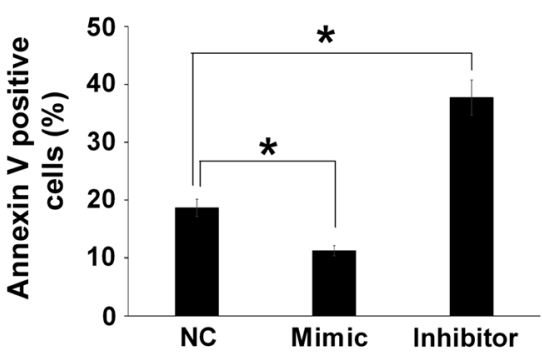

(e)

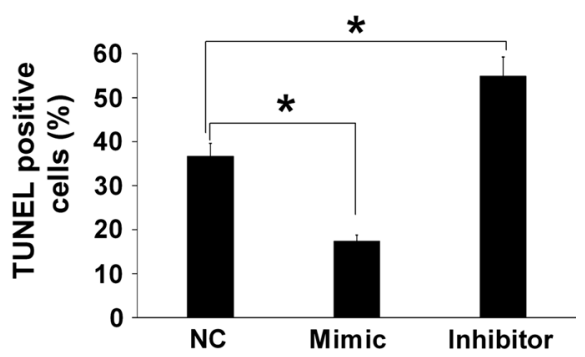

cardiomyocytes treated with miR-499 Mimic or Inhibitor, and then exposed to LPS as above. Red TUNEL-stained positive nuclei, green $\alpha$-actinin antibody staining, blue Hoechst33342-stained nuclei, scale bars $50 \mu \mathrm{m}$. e Quantitative results for apoptotic cells from three independent experiments. A scrambled 22-nt miRNA was used as the negative control (NC). $* p<0.05, * * p<0.01, * * * p<0.001$ compared with negative control (NC) (Color figure online)
siRNAs successfully enhanced or inhibited SOX6 and PDCD4 expression (Fig. S3). In H9c2 cells transfected with SOX6 or PDCD4 overexpressing constructs, the mRNA level of the pro-apoptotic genes $(B A D, B A X, B I D)$ was upregulated, whereas that of the anti-apoptotic gene $B C L-X L$ was downregulated with $1 \mu \mathrm{g} / \mathrm{mL}$ LPS for $6 \mathrm{~h}$ (Fig. 5a). But without LPS stimulation, the transfection of SOX6 or PDCD4 did not affect these genes' expression significantly (Fig. S4). In contrast, the mRNA level of $B A D, B A X$, and $B I D$ was significantly decreased in the $S O X 6$ or $P D C D 4$ knockdown system, whereas that of $B C L$ $X L$ was increased (Fig. 5b).

In LPS-treated rat cardiomyocytes, miR-499 overexpression inhibited Bad, Bax, and Bid mRNA level, and promoted $B c l-x L$ level (Fig. 5c); meanwhile, the expression 
(a)

(c)
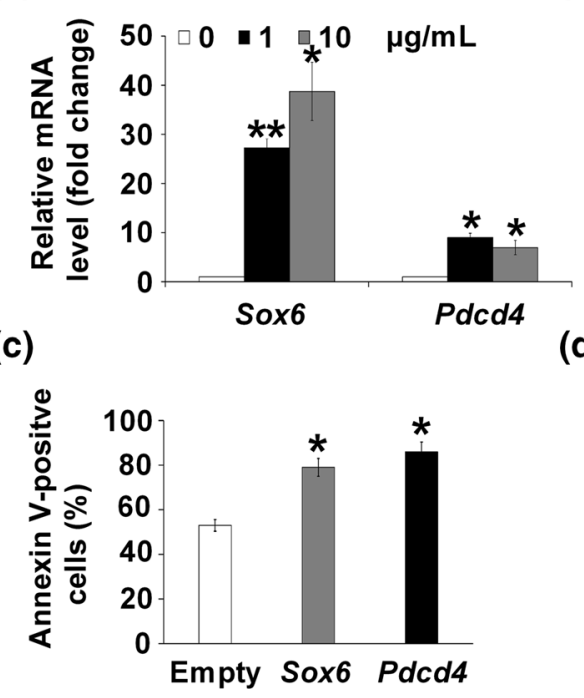

Fig. 3 SOX6 and PDCD4 are involved in LPS-induced cardiac cell apoptosis. a SOX6 and PDCD4 mRNA level in cardiomyocytes exposed to LPS. Cardiomyocytes not treated with LPS $(0 \mu \mathrm{g} / \mathrm{mL})$ were used as the negative control. b SOX6 and PDCD4 protein level in LPS-treated cardiomyocytes. c, d Quantitative flow cytometry results for annexin V/PI-stained cells transfected with SOX6 or (b)

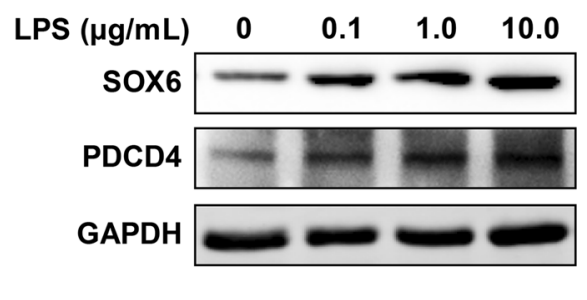

(d)

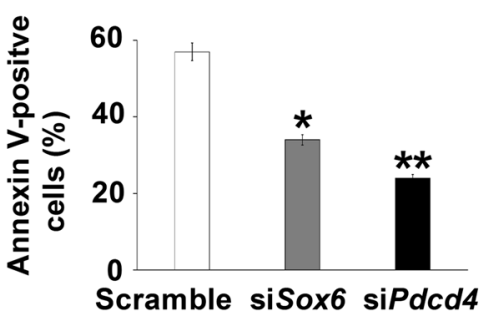

PDCD4 plasmid (c) or with SOX6 siRNA or PDCD4 siRNA (d), respectively, and then exposed to $1 \mu \mathrm{g} / \mathrm{mL}$ LPS for $6 \mathrm{~h}$. The vector plasmid pcDNA3 (Empty) or scrambled siRNA were used as the negative control. $* p<0.05, * * p<0.01$ compared with negative control. Data represent the results of three independent experiments (a)

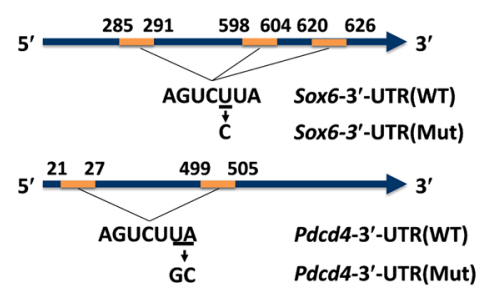

(d)

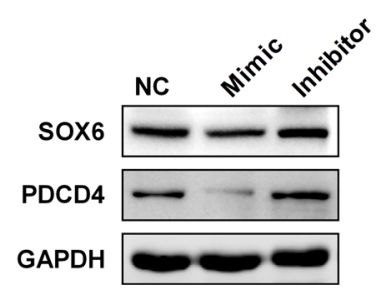

(b)

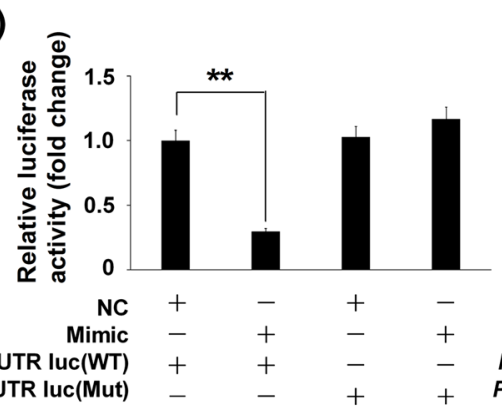

(e)
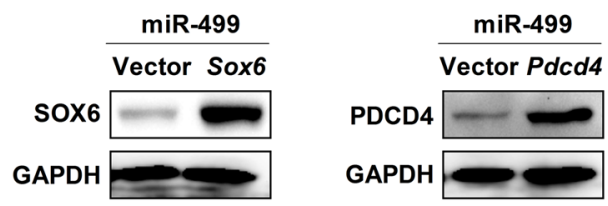

Fig. 4 SOX6 and PDCD4 are targets of miR-499. a Schematic illustration indicates the seed sequences on the $3^{\prime}$-UTR of SOX6 or PDCD4, which are potential target genes of miR-499. The mutant binding sites are underlined. $\mathbf{b}, \mathbf{c}$ Luciferase analysis of the effect of miR-499 on its potential targets. Luciferase activity was measured in HeLa cells cotransfected with miR-499 and SOX6 $3^{\prime}$-UTR luciferase reporter (luc) (wild type [WT]) or SOX6 $3^{\prime}$-UTR luc (mutant [Mut]) (b), PDCD4 3'-UTR luc (WT) or PDCD4 3'-UTR luc (Mut) (c).

of these genes was not affected significantly by miR-499 without LPS treatment (data not shown). In LPS-treated rat cardiomyocytes, the cotransfection of Sox6 or Pdcd4 with experiments (c)

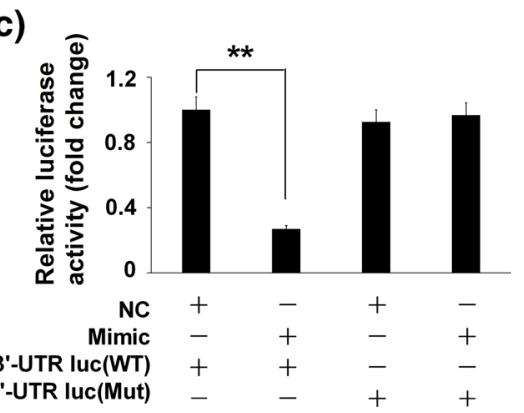

d SOX6 and PDCD4 protein level in cardiomyocytes treated with miR-499 NC, Mimic, or Inhibitor. GAPDH was used as the internal control. e Expression of SOX6 (left) and PDCD4 (right) in H9c2 cells cotransfected with miR-499 Mimic in combination with SOX6 or $P D C D 4$ plasmid, respectively. ${ }^{*} p<0.05,{ }^{* *} p<0.01$ compared with negative control. Data represent the results of three independent

miR-499 reversed the miR-499-mediated cardiac protective effects, which included upregulation of Bad, Bax, and Bid mRNA/protein, and downregulating $B c l-x L$ mRNA/ 
(a)

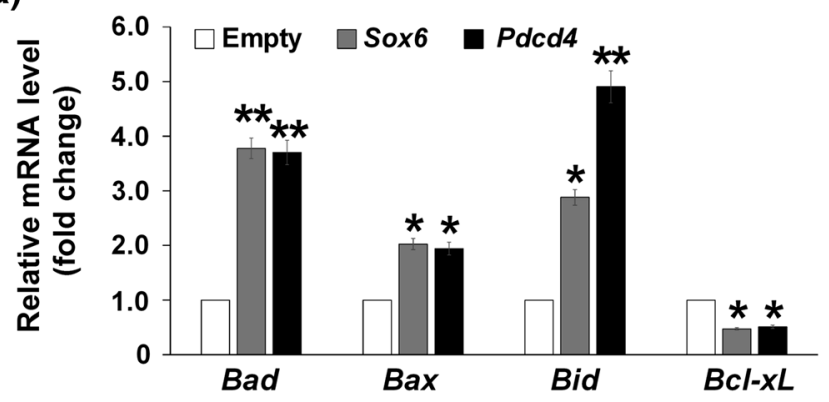

(b)

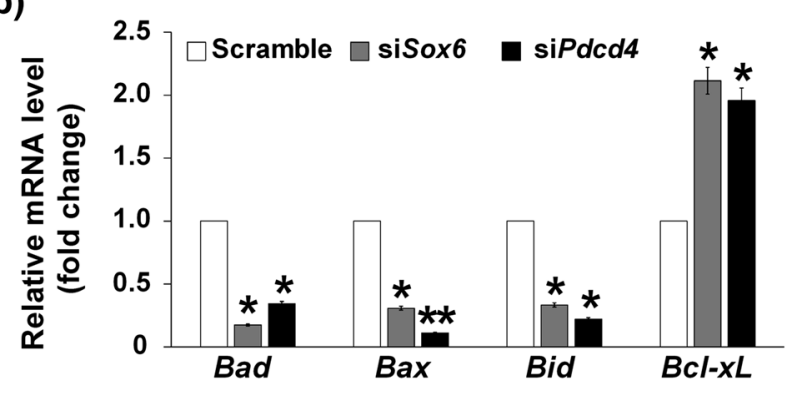

(c)

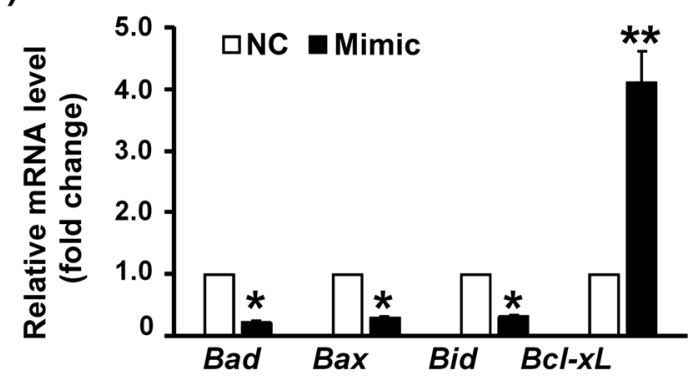

Fig. 5 LPS inhibits miR-499 to activate the SOX6 and PDCD4 pathways. a, b mRNA Level of $B A D, B A X, B I D$, and $B C L-X L$ in $\mathrm{H} 9 \mathrm{c} 2$ cells transfected with $S O X 6$ or PDCD4 plasmid (a) or with $S O X 6$ or $P D C D 4$ siRNA (b), respectively. $\mathbf{c}$ Endogenous mRNA level of $B A D$, $B A X, B I D$, and $B C L-X L$ in response to 6-h stimulation with $1 \mu \mathrm{g} / \mathrm{mL}$ LPS in H9c2 cells transfected with miR-499 NC, Mimic. d, e mRNA Level of $B A D, B A X, B I D$, and $B C L-X L$ in $\mathrm{H} 9 \mathrm{c} 2$ cells cotransfected

protein (Fig. 5d-f), indicating the existence of an miR-499Sox6/Pdcd4-apoptosis pathway.

\section{Discussion}

Cardiomyocyte apoptosis is one of the major pathogenic factors in heart diseases, including septic cardiomyopathy. Bacterial endotoxins, such as LPS, are considered the principal cause of myocardial dysfunction. However, the mechanisms involved are unclear. In this study, we attempted to explore the mechanisms involved in LPS-induced cardiomyocyte apoptosis. We discovered that LPS stimulation reduced miR-499 expression, thereby relieving the inhibitory effect on its target genes SOX6 and PDCD4, (d)

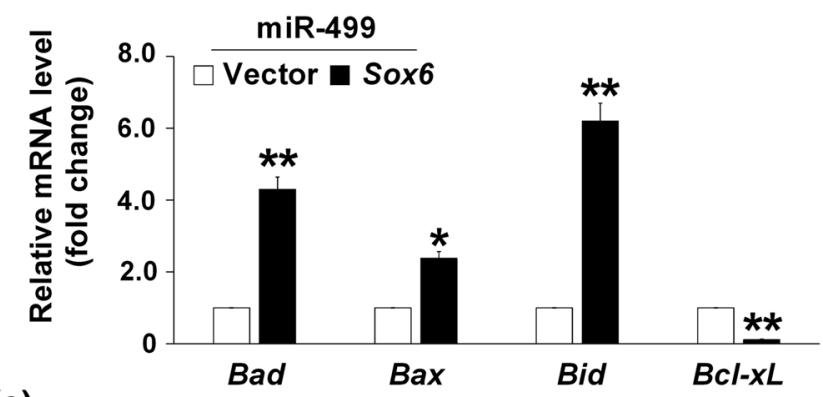

(e)
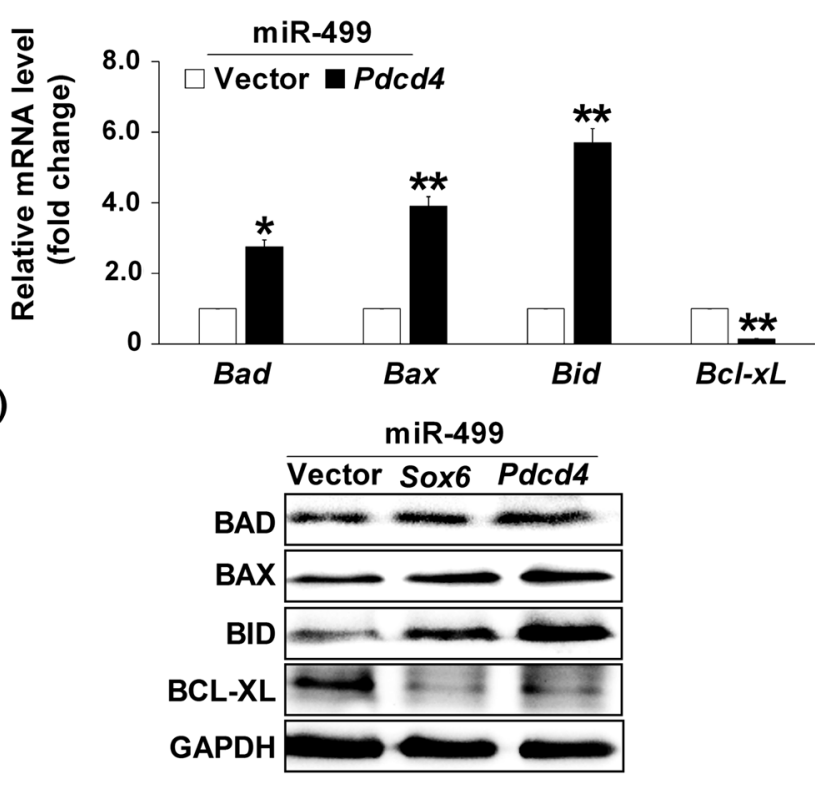

with miR-499 Mimic in combination with SOX6 (d) or PDCD4 (e) plasmid, respectively. f BAD, BAX, BID, and BCL-XL expression in $\mathrm{H} 9 \mathrm{c} 2$ cells cotransfected with miR-499 Mimic in combination with SOX6 or PDCD4 overexpression plasmid, respectively, and then exposed to $1 \mu \mathrm{g} / \mathrm{mL}$ LPS for $6 \mathrm{~h}$. GAPDH was used as the internal control. ${ }^{*} p<0.05, * * p<0.01$ compared with negative control. Data represent the results of three independent experiments

which then activated the BCL-2 family pathway to participate in LPS-induced apoptosis.

SOX6 is a key regulator in cell differentiation and organ development. It also plays important roles in proliferation and apoptosis during stem cell differentiation or cancer progression. In hepatocellular carcinoma, SOX6 activates p21WAF1/CIP1 (p21) expression in a p53-dependent manner, and miR-155 targeting of SOX6 facilitated cell proliferation [11]. In pancreatic $\beta$-cells, SOX6 inhibited cyclin DI promoter activity and negatively regulated cell proliferation by interacting with histone deacetylase 1 (HDAC1) and $\beta$-catenin [33]. SOX6 suppression also caused retinoic acid-dependent apoptosis and blocked neuronal differentiation in the early stages of P19 cell neuronal differentiation [34]. However, the involvement of 
SOX6 in LPS-induced cardiomyocyte apoptosis has not been reported. In our previous research, we found that SOX6, as a repressor of cyclin D1, arrested cardiomyocyte proliferation and facilitated cell cycle exit [10]. The present study is the first demonstration, to our knowledge, that SOX6 expression is elevated in LPS-treated cardiomyocytes in a time- and dose-dependent manner, and the upregulated SOX6 enhances LPS-induced apoptosis.

We also found that PDCD4 was upregulated in LPStreated cardiomyocytes. PDCD4 is a tumor repressor, suppressing tumor growth through different mechanisms [35]. PDCD4 also plays an important role in various inflammatory diseases. $P D C D 4$-deficient cells were significantly less sensitive to apoptosis, and PDCD4 overexpression in $\beta$-TC-6 cells increased their susceptibility to TNF- $\alpha$ plus IFN- $\gamma$-induced apoptosis [28]. PDCD4 promoted activation of the transcription factor nuclear factor$\kappa \mathrm{B}(\mathrm{NF}-\kappa \mathrm{B})$ and suppressed IL-10; Pdcd4-deficient mice were less susceptible to LPS-induced death, suggesting PDCD4 is a proinflammatory factor [25]. However, other reports showed that PDCD4 deficiency increased TNF- $\alpha$ protein expression in LPS-treated RAW264.7 macrophages, indicating that PDCD4 is an anti-inflammatory factor [36, 37]. Therefore, the exact role of PDCD4 in inflammatory diseases remains to be investigated. It should be noted that the effect of LPS stimulation on PDCD4 expression differs between reports. In primary bone marrow-derived macrophages and RAW264.7 macrophages, PDCD4 was upregulated at $1 \mathrm{~h}$ after LPS treatment and gradually decreased at $6 \mathrm{~h}$ [24]. Another report showed that PDCD4 remained at a high level at $8 \mathrm{~h}$ after LPS stimulation in human peripheral blood mononuclear cells [25]. It is easy to understand that dynamic feedback is involved in the response to various stimuli; any difference could lead to different feedback. Nevertheless, we found that PDCD4 was upregulated in LPS-stimulated cardiomyocytes.

To understand how SOX6 and PDCD4 expression is regulated in LPS-treated cardiomyocytes, we detected the expression of a series of miRNAs. MiR-499 and miR-21 were downregulated in LPS-induced cells in a dose- and time-dependent manner, while there was no significant change to miR-1, miR-133, and miR-208 expression. A growing amount of evidence demonstrates that miRNAs can regulate apoptosis [38]. Thirteen miRNAs, including miR-21, were upregulated in early-stage dilated cardiomyopathy, whereas 11 miRNAs including miR-499 were downregulated [39]. MiR-499 was also downregulated in cardiomyocytes exposed to anoxia [40]. In our previous work, we showed that miR-499 and miR-21 were upregulated in $\mathrm{H}_{2} \mathrm{O}_{2}$-induced cardiomyocyte apoptosis [21]. Hence, miRNA expression is correlated with various diseases, stimuli, cells, and detection time [41]. Several reports demonstrated that transient transfection of miR-1 and miR-499 reduced proliferation and enhanced differentiation into cardiomyocytes in human cardiac progenitor cells and embryonic stem cells [6]. In a transgenic mouse model, elevated miR-499 levels affected Egrl and Fos, the immediate early genes in the response to cardiac stress [31]. Additionally, in rat myocardial infarction area induced by anoxia and ischemia, miR-499 inhibited cardiomyocyte apoptosis through regulation of mitochondrial dynamics by targeting calcineurin and dynamin-related protein-1 [40].

In the present study, LPS stimulation suppressed miR499 expression, which led to elevation of the cardiomyocyte apoptosis rate. Our experiments also showed that the BCL-2 family is involved in miR-499-SOX6-PDCD4 apoptotic regulation. MiR-499 inhibited the expression of pro-apoptotic genes and upregulated expression of the antiapoptotic gene $B C L-X L$. SOX6 and PDCD4 reversed these effects, suggesting that miR-499 regulates $B A D, B A X, B I D$, and $B C L-X L$ expression by inhibiting $S O X 6$ and $P D C D 4$, thereby playing a key role in LPS-induced cardiomyocyte apoptosis. To investigate how LPS downregulates miR499 , sequence analysis of the promoter region of $M Y H 7 B$, the host gene of miR-499, was performed. The result showed that it contains one $\mathrm{NF}-\kappa \mathrm{B}$ binding site, which is conserved among rats, mice, and humans. NF- $\kappa \mathrm{B}$ is activated by LPS-induced TNF- $\alpha$ [2]. However, when we transfected the luciferase reporter construct containing a 1000-bp region of the $M Y H 7 B$ regulatory fragment upstream of the translational start site into $\mathrm{H} 9 \mathrm{c} 2$ cells that were then treated with LPS, no significant change in luciferase activity was observed after LPS treatment (data not shown). It should be noticed that we used H9c2 cells for the most experiments to demonstrate SOX6 and PDCD4 as the targets of miR-499. Although this cell line is different from primary neonatal myocytes, it is commonly used as a cardiomyocyte model for mechanism investigation [42, 43]. However, further investigation is required to clarify the upstream regulation mechanism of miR-499. In summary, LPS stimulation relieved the inhibitory effect of miR-499 on its target genes SOX6 and PDCD4, which enhanced LPS-induced cardiomyocyte apoptosis through the BCL-2 family members. Overexpression of miR-499 protected cardiomyocytes against LPS-induced apoptosis.

Acknowledgments This work was supported by the National Natural Science Foundation of China $(81371889$, 81370236, 81170713, 81472022), the Natural Science Foundation of Beijing, China (5122021), the Leading Academic Discipline Project of Beijing Education Bureau, the 111 Project of China (B07001), and the Collaborative Research Project of PUHSC and National Taiwan University (BMU20120315).

\section{Compliance with ethical standards}

Conflict of interests The authors declare no conflict of interest. 
Open Access This article is distributed under the terms of the Creative Commons Attribution 4.0 International License (http://crea tivecommons.org/licenses/by/4.0/), which permits unrestricted use, distribution, and reproduction in any medium, provided you give appropriate credit to the original author(s) and the source, provide a link to the Creative Commons license, and indicate if changes were made.

\section{References}

1. Romero-Bermejo FJ, Ruiz-Bailen M, Gil-Cebrian J, HuertosRanchal MJ (2011) Sepsis-induced cardiomyopathy. Curr Cardiol Rev 7:163-183

2. Russell JA, Boyd J, Nakada T, Thair S, Walley KR (2011) Molecular mechanisms of sepsis. Contrib Microbiol 17:48-85

3. Marchant DJ, Boyd JH, Lin DC, Granville DJ, Garmaroudi FS, McManus BM (2012) Inflammation in myocardial diseases. Circ Res 110:126-144

4. Hagiwara N (2011) Sox6, jack of all trades: a versatile regulatory protein in vertebrate development. Dev Dyn 240:1311-1321

5. Yi Z, Cohen-Barak O, Hagiwara N, Kingsley PD, Fuchs DA, Erickson DT, Epner EM, Palis J, Brilliant MH (2006) Sox6 directly silences epsilon globin expression in definitive erythropoiesis. PLoS Genet 2:e14

6. Sluijter JP, van Mil A, van Vliet P, Metz CH, Liu J, Doevendans PA, Goumans MJ (2010) MicroRNA-1 and -499 regulate differentiation and proliferation in human-derived cardiomyocyte progenitor cells. Arterioscler Thromb Vasc Biol 30:859-868

7. Cohen-Barak O, Yi Z, Hagiwara N, Monzen K, Komuro I, Brilliant MH (2003) Sox6 regulation of cardiac myocyte development. Nucleic Acids Res 31:5941-5948

8. Li H, Zheng D, Zhang B, Liu L, Ou J, Chen W, Xiong S, Gu Y, Yang J (2014) Mir-208 promotes cell proliferation by repressing SOX6 expression in human esophageal squamous cell carcinoma. J Transl Med 12:196

9. Zhu Y, Xia Y, Niu H, Chen Y (2014) MiR-16 induced the suppression of cell apoptosis while promote proliferation in esophageal squamous cell carcinoma. Cell Physiol Biochem 33:1340-1348

10. Li X, Wang J, Jia Z, Cui Q, Zhang C, Wang W, Chen P, Ma K, Zhou C (2013) MiR-499 regulates cell proliferation and apoptosis during late-stage cardiac differentiation via Sox6 and cyclin D1. PLoS One 8:e74504

11. Xie Q, Chen X, Lu F, Zhang T, Hao M, Wang Y, Zhao J, McCrae MA, Zhuang H (2012) Aberrant expression of microRNA 155 may accelerate cell proliferation by targeting sex-determining region $\mathrm{Y}$ box 6 in hepatocellular carcinoma. Cancer 118:2431-2442

12. Scalbert E, Bril A (2008) Implication of microRNAs in the cardiovascular system. Curr Opin Pharmacol 8:181-188

13. Condorelli G, Latronico MV, Cavarretta E (2014) microRNAs in cardiovascular diseases: current knowledge and the road ahead. J Am Coll Cardiol 63:2177-2187

14. Boon RA, Dimmeler S (2014) MicroRNAs in myocardial infarction. Nat Rev Cardiol 12:135-142

15. Wang GK, Zhu JQ, Zhang JT, Li Q, Li Y, He J, Qin YW, Jing Q (2010) Circulating microRNA: a novel potential biomarker for early diagnosis of acute myocardial infarction in humans. Eur Heart J 31:659-666

16. Ai J, Zhang R, Li Y, Pu J, Lu Y, Jiao J, Li K, Yu B, Li Z, Wang R, Wang L, Li Q, Wang N, Shan H, Yang B (2010) Circulating microRNA-1 as a potential novel biomarker for acute myocardial infarction. Biochem Biophys Res Commun 391:73-77
17. Adachi T, Nakanishi M, Otsuka Y, Nishimura K, Hirokawa G, Goto Y, Nonogi H, Iwai N (2010) Plasma microRNA 499 as a biomarker of acute myocardial infarction. Clin Chem 56:1183-1185

18. van Rooij E, Quiat D, Johnson BA, Sutherland LB, Qi X, Richardson JA, Kelm RJ Jr, Olson EN (2009) A family of microRNAs encoded by myosin genes governs myosin expression and muscle performance. Dev Cell 17:662-673

19. Fu JD, Rushing SN, Lieu DK, Chan CW, Kong CW, Geng L, Wilson KD, Chiamvimonvat N, Boheler KR, Wu JC, Keller G, Hajjar RJ, Li RA (2011) Distinct roles of microRNA-1 and -499 in ventricular specification and functional maturation of human embryonic stem cell-derived cardiomyocytes. PLoS One 6:e27417

20. Wilson KD, Hu S, Venkatasubrahmanyam S, Fu JD, Sun N, Abilez OJ, Baugh JJ, Jia F, Ghosh Z, Li RA, Butte AJ, Wu JC (2010) Dynamic microRNA expression programs during cardiac differentiation of human embryonic stem cells: role for miR-499. Circ Cardiovasc Genet 3:426-435

21. Wang J, Jia Z, Zhang C, Sun M, Wang W, Chen P, Ma K, Zhang Y, Li X, Zhou C (2014) miR-499 protects cardiomyocytes from $\mathrm{H}_{2} \mathrm{O}_{2}$-induced apoptosis via its effects on Pdcd4 and Pacs2. RNA Biol 11:339-350

22. Shibahara K, Asano M, Ishida Y, Aoki T, Koike T, Honjo T (1995) Isolation of a novel mouse gene MA-3 that is induced upon programmed cell death. Gene 166:297-301

23. Lankat-Buttgereit B, Goke R (2009) The tumour suppressor Pdcd4: recent advances in the elucidation of function and regulation. Biol Cell 101:309-317

24. van den Bosch MW, Palsson-Mcdermott E, Johnson DS, O’Neill LA (2014) LPS induces the degradation of programmed cell death protein 4 (PDCD4) to release Twist2, activating c-Maf transcription to promote interleukin-10 production. J Biol Chem 289:22980-22990

25. Sheedy FJ, Palsson-McDermott E, Hennessy EJ, Martin C, O'Leary JJ, Ruan Q, Johnson DS, Chen Y, O'Neill LA (2010) Negative regulation of TLR4 via targeting of the proinflammatory tumor suppressor PDCD4 by the microRNA miR-21. Nat Immunol 11:141-147

26. Cash AC, Andrews J (2012) Fine scale analysis of gene expression in Drosophila melanogaster gonads reveals Programmed cell death 4 promotes the differentiation of female germline stem cells. BMC Dev Biol 12:4

27. Lo Furno D, Graziano AC, Caggia S, Perrotta RE, Tarico MS, Giuffrida R, Cardile V (2013) Decrease of apoptosis markers during adipogenic differentiation of mesenchymal stem cells from human adipose tissue. Apoptosis 18:578-588

28. Ruan Q, Wang T, Kameswaran V, Wei Q, Johnson DS, Matschinsky F, Shi W, Chen YH (2011) The microRNA-21PDCD4 axis prevents type 1 diabetes by blocking pancreatic beta cell death. Proc Natl Acad Sci USA 108:12030-12035

29. Yang M, Liu R, Li X, Liao J, Pu Y, Pan E, Yin L, Wang Y (2014) miRNA-183 suppresses apoptosis and promotes proliferation in esophageal cancer by targeting PDCD4. Mol Cells 37:873-880

30. Liu J, Wu LL, Li L, Zhang L, Song ZE (2005) Growth-promoting effect of platelet-derived growth factor on rat cardiac myocytes. Regul Pept 127:11-18

31. Shieh JT, Huang Y, Gilmore J, Srivastava D (2011) Elevated miR-499 levels blunt the cardiac stress response. PLoS One 6:e19481

32. Cheng EH, Wei MC, Weiler S, Flavell RA, Mak TW, Lindsten T, Korsmeyer SJ (2001) BCL-2, BCL-X(L) sequester BH3 domainonly molecules preventing BAX- and BAK-mediated mitochondrial apoptosis. Mol Cell 8:705-711

33. Iguchi $\mathrm{H}$, Urashima $\mathrm{Y}$, Inagaki $\mathrm{Y}$, Ikeda $\mathrm{Y}$, Okamura M, Tanaka T, Uchida A, Yamamoto TT, Kodama T, Sakai J (2007) SOX6 suppresses cyclin D1 promoter activity by interacting with beta- 
catenin and histone deacetylase 1, and its down-regulation induces pancreatic beta-cell proliferation. J Biol Chem 282:19052-19061

34. Hamada-Kanazawa M, Ishikawa K, Ogawa D, Kanai M, Kawai Y, Narahara M, Miyake M (2004) Suppression of Sox6 in P19 cells leads to failure of neuronal differentiation by retinoic acid and induces retinoic acid-dependent apoptosis. FEBS Lett 577:60-66

35. Matsuhashi S, Hamajima H, Xia J, Zhang H, Mizuta T, Anzai K, Ozaki I (2014) Control of a tumor suppressor PDCD4: degradation mechanisms of the protein in hepatocellular carcinoma cells. Cell Signal 26:603-610

36. Yasuda M, Schmid T, Rubsamen D, Colburn NH, Irie K, Murakami A (2010) Downregulation of programmed cell death 4 by inflammatory conditions contributes to the generation of the tumor promoting microenvironment. Mol Carcinog 49:837-848

37. Wang X, Zhang L, Wei Z, Zhang X, Gao Q, Ma Y, Liu X, Jiang Y, Liu X, Guo C, Wang X (2013) The inhibitory action of PDCD4 in lipopolysaccharide/D-galactosamine-induced acute liver injury. Lab Invest 93:291-302

38. Li P (2010) MicroRNAs in cardiac apoptosis. J Cardiovasc Transl Res 3:219-224
39. Isserlin R, Merico D, Wang D, Vuckovic D, Bousette N, Gramolini AO, Bader GD, Emili A (2015) Systems analysis reveals down-regulation of a network of pro-survival miRNAs drives the apoptotic response in dilated cardiomyopathy. Mol BioSyst $11: 239-251$

40. Wang JX, Jiao JQ, Li Q, Long B, Wang K, Liu JP, Li YR, Li PF (2011) miR-499 regulates mitochondrial dynamics by targeting calcineurin and dynamin-related protein-1. Nat Med 17:71-78

41. Ye Y, Perez-Polo JR, Qian J, Birnbaum Y (2011) The role of microRNA in modulating myocardial ischemia-reperfusion injury. Physiol Genomics 43:534-542

42. Lage R, Moscoso I, Fernández-Trasancos Á, Cebro M, Couselo M, Fandiño-Vaquero R, Bravo SB, Sierra J, González-Juanatey JR, Eiras S (2015) Differential behaviour of epicardial adipose tissue-secretomes with high and low orosomucoid levels from patients with cardiovascular disease in $\mathrm{H} 9 \mathrm{C} 2$ cells. Mol Cell Endocrinol 416:77-87

43. Sangeetha KN, Lakshmi BS, Niranjali Devaraj S (2015) Dexamethasone promotes hypertrophy of $\mathrm{H} 9 \mathrm{C} 2$ cardiomyocytes through calcineurin B pathway, independent of NFAT activation. Mol Cell Biochem. doi:10.1007/s11010-015-2586-9 